Résumés des conférences et travaux

\title{
Art et Archéologie de la Chine pré-impériale
}

\section{Alain Thote}

\section{OpenEdition \\ Journals}

Édition électronique

URL : https://journals.openedition.org/ashp/1212

DOI : 10.4000/ashp.1212

ISSN : 1969-6310

Éditeur

Publications de l'École Pratique des Hautes Études

\section{Édition imprimée}

Date de publication : 1 octobre 2011

Pagination : 247-249

ISSN : 0766-0677

\section{Référence électronique}

Alain Thote, "Art et Archéologie de la Chine pré-impériale ", Annuaire de l'École pratique des hautes études (EPHE), Section des sciences historiques et philologiques [En ligne], 142 | 2011, mis en ligne le 27 juillet 2011, consulté le 06 juillet 2021. URL : http://journals.openedition.org/ashp/1212 ; DOI : https:// doi.org/10.4000/ashp.1212 


\title{
ART ET ARCHÉOLOGIE DE LA CHINE PRÉ-IMPÉR I A LE
}

\author{
Directeur d'études : M. Alain Thоте, \\ correspondant de l'Institut
}

Programme de l'année 2009-2010 : Études sur la culture matérielle des Zhou : le miroir.

En général, les recherches sur les miroirs chinois anciens tiennent insuffisamment compte du contexte des découvertes, plaçant sur le même plan les objets de fouilles et les pièces de collection. De loin les plus nombreuses, ces dernières ne présentent pas cependant un grand intérêt et les faux pullulent. D'étude en étude sont reproduits les mêmes présupposés, les mêmes erreurs de datation. On a donc souhaité aborder la question en privilégiant les découvertes archéologiques.

Le dépôt de miroirs dans les tombes chinoises est souvent considéré comme une pratique de bon augure. De fait, le caractère magique de ces objets est attesté de plusieurs façons, par leurs inscriptions, leurs motifs, et parfois leur emplacement. Ainsi, dans leur décor, les références à la cosmologie sont nombreuses sous les Han (206 av.220 apr. J.-C.). Au début de la dynastie, elles restent allusives, puis s'affirment dans la seconde moitié du $\mathrm{II}^{\mathrm{e}} \mathrm{s}$. av. J.-C. grâce à l'introduction d'un système complexe de correspondances où interviennent le ciel et la terre, le soleil et la lune, les quatre animaux des orients, les douze animaux du zodiaque, les huit trigrammes, les vingt-huit mansions de la lune, les cinq éléments (métal, feu, bois, eau, terre), le yin et le yang. D'autre part, il existe une variété de miroirs dits TLV parce qu'ornés de motifs en T, en $\mathrm{L}$ et en V. Des liens subtils se tissent entre le décor de ces miroirs et deux autres objets : les astrolabes utilisés pour la divination et la table du jeu de liubo 六博, dont on ne connaît plus les règles aujourd'hui mais qui est aussi gravée de motifs T, L et V. L'article de Lillian Lan-ying Tseng, « Representation and Appropriation: Rethinking the TLV Mirror in Han China » dans Early China, 29 (2004), p. 163-215, nous a servi de guide pour étudier ces liens (il s'agit d'une des plus récentes études d'une très longue série sur le sujet). En reproduisant dans le schéma TLV une table de divination, le miroir acquiert une fonction de même nature, conjurer le mauvais sort (去不祥). La présence d'immortels, de Xi Wang Mu 西王母 (la reine mère de l'Ouest), de souhaits de longévité, renforce ce jeu d'associations positives, et donc son rôle religieux.

Le cas des trois miroirs déposés dans la tombe de Dou Wan 窦綰, une princesse morte un peu avant 104 av. J.-C., révèle cependant l'ambivalence des usages du miroir, à la fois objet du quotidien et talisman. L'un d'eux était rangé dans un coffre tandis qu'un autre (du type «TLV ») faisait partie d'un nécessaire de toilette dans une boîte en laque déposée à l'intérieur du cercueil, selon une tradition déjà en cours un siècle plus tôt. Il portait l'inscription d'un vœu de bonheur et de longévité (pour ici-bas et dans l'au-delà). Enfin le dernier, miniature et donc sans autre fonction que magique, se trouvait placé dans la main de la défunte. 
Le décor de plusieurs miroirs Han est composé de manière rayonnante depuis le centre, en suivant les axes de deux diagonales. Le carré central où se trouve l'anneau de suspension est entouré de cercles concentriques qui définissent des registres. Les motifs sont disposés ainsi en progression numérique du centre vers la périphérie, de 4 à 8 , puis à 12 , enfin à 16 , allant parfois jusqu'à atteindre 24 . L'analyse en série des inscriptions a aussi permis de dégager plusieurs thèmes récurrents : 1) présence de vœux de longévité perpétuée par une descendance nombreuse, souhaits de prospérité, de nourriture et boisson à satiété, d'honneurs, de bonheur sans fin;2) allusions à la lumière et à l'éclat du soleil en liaison avec le pouvoir réfléchissant du miroir; 3) évocation de la séparation provoquée par un voyage au loin et sans doute, à travers la métaphore du voyage, par la mort d'un époux ou d'une épouse; 4) excellence des alliages utilisés pour fondre des miroirs de qualité.

Les inscriptions, dont le contenu est homogène, le nombre limité de motifs et enfin la standardisation des décors ont tous les trois contribué à diffuser une idéologie, à uniformiser un système de croyances sur un très vaste territoire. Cette diffusion est descendue des meilleurs ateliers de l'empire pour être relayée par les ateliers provinciaux - des différences de qualité sont sensibles entre les premiers et les seconds. D'un objet modeste par ses dimensions, le miroir est devenu un concentré des aspirations, des croyances, des associations symboliques Han.

Durant la longue période de gestation qui précéda son soudain développement à la toute fin des Royaumes combattants, rien ne laisse supposer qu'il concentrerait de telles associations. C'est ce qu'a montré la seconde partie de la conférence consacrée aux origines du miroir en Chine, à ses liens avec les cultures périphériques de l'Ouest et du Nord, et à sa diffusion. Plusieurs points ont été abordés, et en premier lieu la question des miroirs d'Anyang 安陽, la capitale Shang aux XIII'-XI ${ }^{\mathrm{e}}$ s. av. J.-C. Originaires probablement de la culture beaucoup plus ancienne de Qijia 齊家 (env. 26002000 av. J.-C.), ils ont été fondus selon une technique différente de celle des Shang, ce qui confirme le caractère exogène de leur fabrication. À Anyang, rien n'indique que l'introduction de miroirs dans la culture Shang n'ait entraîné l'apparition d'une production locale.

Plus tard, sous les Zhou occidentaux, les miroirs sont petits (entre 5 et $7 \mathrm{~cm}$ de diamètre en moyenne, $9 \mathrm{~cm}$ maximum), en général sans décor, la fonte en est médiocre. Certains ont une face réfléchissante concave, ce qui conduit à les identifier comme des yangsui 陽燧 (ou 隧), 《 miroirs pour obtenir le feu pur », selon la traduction de Pelliot, qui se base sur des textes plus tardifs, principalement bouddhiques. À l'exception d'un miroir provenant d'une tombe princière de Guo 虢, quand ils ont un décor, celui-ci est exécuté dans une technique assez sommaire, limitée à un mince filet en relief (le décor a donc été gravé directement sur la paroi du moule). Ce ne sont donc pas non plus les ateliers métropolitains des vallées de la Wei et du moyen fleuve Jaune qui les ont fondus.

Les premiers miroirs et yangsui décorés découverts en Chine centrale attestent en fait de liens très forts avec l'art steppique, avec par exemple l'image d'un rapace en vol ou une scène de prédation. Des rapprochements ont pu être faits avec des appliques et miroirs à décor d'oiseaux de proie de l'Altaï ou du Xinjiang, avec des plaques de la culture de Xiajiadian supérieur 夏家店 (début du premier millénaire avant notre ère), 
des pétroglyphes de la Mongolie intérieure, des objets de parure et des couteaux de la région des Ordos (culture de Maoqinggou 毛慶溝, VII ${ }^{\mathrm{e}}-\mathrm{V}^{\mathrm{e}}$ S. av. J.-C.). Ainsi, le corpus que nous avons établi des pièces découvertes en contexte chinois, jusqu'au $\mathrm{VI}^{\mathrm{e}}$ s. av. J.-C., oriente toujours les comparaisons vers des objets provenant du monde steppique.

Dans la principauté de Jin 晋, les ateliers de Houma 侯马 (Shanxi) deviennent aux $\mathrm{VI}^{\mathrm{e}}$ et $\mathrm{V}^{\mathrm{e}} \mathrm{s}$. av. J.-C. le premier centre de production, tant par la quantité que par la qualité des pièces. Deux grands styles s'y développent. L'un s'apparente au style des bronzes rituels issus du même centre métallurgique. L'autre est une forme sinisée du style animalier des steppes. Aussi bien le rendu naturaliste de quelques animaux, le choix d'espèces rarement représentées sur les bronzes rituels (sangliers, par exemple) que la relation prédateur/proie rappellent avec nuance, comme en écho, les origines non-chinoises du miroir. Cette combinaison de styles caractérise bien les ateliers de Houma. Ces derniers devaient passer le relais aux ateliers du royaume de Chu qui furent à leur suite les plus novateurs dans la production des miroirs.

Comme les conférences n'ont pu se tenir que sur un seul semestre, le temps nous a manqué pour examiner dans le détail la production de Chu, florissante au $\mathrm{III}^{\mathrm{e}} \mathrm{s}$. av. J.-C. Cependant, nous avons pu établir quelques points. Notamment, les miroirs déposés dans les tombes font toujours partie de nécessaires de toilette, aussi bien pour les hommes que les femmes. Mais entre Jin et Chu une différence importante est à noter : les tombes aristocratiques de Jin renferment souvent des miroirs, tandis qu'à Chu la possession d'un miroir ne semble pas être l'apanage de personnes ayant un statut social élevé. D'autre part, rien dans le décor de tous ces miroirs, qu'ils soient de Jin ou de $\mathrm{Chu}$, ne laisse supposer une quelconque relation avec les thématiques qui devaient dominer plus tard sous les Han, telles que la cosmologie ou les motifs de bon augure. Cependant, les miroirs de Chu sont ornés d'une façon qui ne doit rien à l'art traditionnel du bronze. Les compositions sont inspirées de décors peints ou, plus souvent, brodés. Deux ou trois registres de motifs se superposent et parfois s'interpénètrent, créant une illusion de profondeur entre les différents plans du décor - tandis que la face principale du miroir permet d'obtenir d'une autre façon un effet comparable grâce à son pouvoir réfléchissant.

Une séance a été consacrée à un rapport sur un colloque auquel avait été invité le directeur d'études, « The Sarcophagus East and West » (Institute for the Study of the Ancient World, New York University, 2-3 octobre 2009), permettant de confronter deux conceptions différentes des cercueils et sarcophages dans les mondes grécoromain d'une part et chinois d'autre part. Lors d'une autre séance, $\mathrm{M}^{\mathrm{me}}$ Chen Chaorong, chercheur à l'Institut d'histoire et de philologie de l'Academia Sinica, Taipei, invitée par l'École française d'Extrême-Orient, est venue présenter son programme de numérisation des inscriptions sur bronze. 